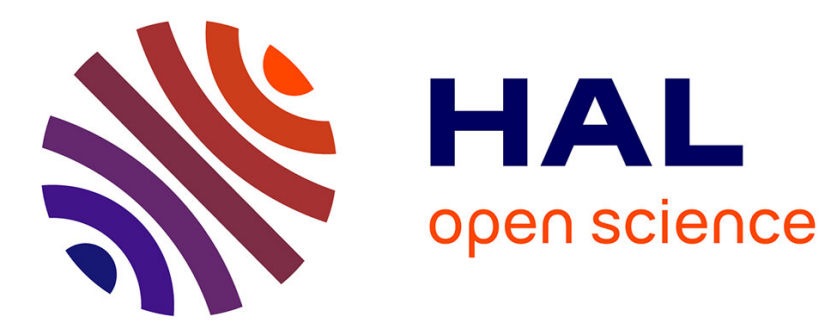

\title{
DUX4 Pathological Expression: Causes and Consequences in Cancer
}

Carla Dib, Vlada Zakharova, Ekaterina Popova, Ekaterina Kiseleva, Boris Chernyak, Marc Lipinski, Yegor Vassetzky

\section{To cite this version:}

Carla Dib, Vlada Zakharova, Ekaterina Popova, Ekaterina Kiseleva, Boris Chernyak, et al.. DUX4 Pathological Expression: Causes and Consequences in Cancer. Trends in Cancer, 2019, 5 (5), pp.268271. 10.1016/j.trecan.2019.03.001 . hal-02323059

\section{HAL Id: hal-02323059 \\ https://hal.science/hal-02323059}

Submitted on 25 Oct 2021

HAL is a multi-disciplinary open access archive for the deposit and dissemination of scientific research documents, whether they are published or not. The documents may come from teaching and research institutions in France or abroad, or from public or private research centers.
L'archive ouverte pluridisciplinaire HAL, est destinée au dépôt et à la diffusion de documents scientifiques de niveau recherche, publiés ou non, émanant des établissements d'enseignement et de recherche français ou étrangers, des laboratoires publics ou privés.

\section{다)(1) $(5$}

Distributed under a Creative Commons Attribution - NonCommercial| 4.0 International 


\section{DUX4 pathological expression: causes and consequences in cancer}

Carla Dib ${ }^{1,2}$, Vlada Zakharova ${ }^{1,2,4}$, Ekaterina Popova ${ }^{3}$, Ekaterina Kiseleva $^{5}$, Boris Chernyak ${ }^{3}$, Marc Lipinski $^{1,2}$, Yegor S. Vassetzky ${ }^{1,2,5,6}$

${ }^{1}$ UMR 8126, CNRS, Univ. Paris-Sud, Université Paris Saclay, Institut Gustave Roussy, F-94805 Villejuif, France

${ }^{2}$ LIA 1066 LFR2O French-Russian Joint Cancer Research Laboratory, 94805 Villejuif,

France

${ }^{3}$ Belozersky Institute of Physico-Chemical Biology, Lomonosov Moscow State University, 119991 Moscow, Russia

${ }^{4}$ CNRS UMR7216 "Epigénétique et Destin Cellulaire" Université Paris Diderot Paris 7

${ }^{5}$ Koltzov Institute of Developmental Biology, Moscow 117334, Russia

${ }^{6}$ To whom correspondence should be addressed:

Yegor S. Vassetzky,

CNRS UMR8126,

Institut Gustave Roussy,

F-94805 Villejuif, France

Tel: +(33)142116283 ;

E-mail: vassetzky@igr.fr

Keywords: DUX4, Leukaemia, Sarcoma, FSHD

Word count: 1160 


\begin{abstract}
DUX4, a double homeobox transcription factor, has been mostly studied in facioscapulohumeral dystrophy (FSHD), a pathology linked to a deletion of subtelomeric repeats on chromosome 4q. More recently, however, the gene has been associated with various sarcomas and hematological malignancies. Drugs developed for FSHD could be tested on cancer cells to develop efficient treatment strategies for both pathologies.
\end{abstract}


DUX4 is a double homeobox transcription factor encoded within the D4Z4 subtelomeric repeat element on chromosome 4q. Recently, DUX4 rearrangements were reported in a frequent paediatric subtype of B-cell precursor acute lymphoblastic leukaemia (BCP-ALL) (reviewed in [1]), in Ewinglike sarcoma [2] and rhabdomyosarcoma (RMS) [3]. Previously, aberrant expression of DUX4 was identified as a major factor in the aetiology of facioscapulohumeral dystrophy (FSHD), an autosomal dominant disorder. Below, we discuss features and consequences of DUX4 gene rearrangements in malignancies and new therapeutic approaches in the context of FSHD that might prove useful for cancer treatment.

\section{$D U X 4$ expression and gene rearrangements}

In humans, an aberrantly expressed DUX4 has been observed in numerous malignancies including renal, breast and testicular cancers (source: Human Protein Atlas ${ }^{\mathrm{i}}$ ). Translocations $(4 ; 19)$ have been observed in Ewing-like sarcomas resulting in portions of DUX4 fused with partner genes. One translocation produced a fused protein associating the N-terminal part of CIC, an ETS family transcription factor, and the C-terminus of DUX4 (Figure 1A) resulting in a dysregulation of the transcriptional activity of the fused gene [2]. In embryonic rhabdomyosarcoma (RMS), a t(4;22) rearrangement led to the production of an EWSR1-DUX4 chimeric protein [3]. Other DUX4 rearrangements included the insertion of a truncated copy of DUX4 into either an intron of ERG [4] or the $I G H$ gene locus [1] (Figure 1B,C), characteristic of a subtype of BCP-ALL. Both chimeric proteins had a DUX4 C-terminal truncation. Additionally, some DUX4 C-terminal aminoacids have been found replaced by aminoacids encoded by non-coding parts of the partner gene.

\section{$D U X 4$ regulation in normal and pathological conditions}

Most data concerning the function and regulation of DUX4 were generated from studies in FSHD [5]. The subtelomeric D4Z4 repeat has long been considered as "junk" DNA until DUX4 transcripts were discovered in FSHD muscle cells following the identification of an open reading frame (ORF) encoding two homeoboxes [6]. DUX4 expression is controlled by two DUX4-specific enhancers and an insulator proximal to the D4Z4 repeat [7]. Epigenetic modifications including DNA hypomethylation of D4Z4 units reported in FSHD patients appear to correlate with the severity of the disease [8]. Non-coding RNAs, miRNAs, telomere shortening, and long-range chromatin interactions also affect the expression of DUX4. In BCP-ALL, following the translocation, DUX4 transcription is regulated by control elements in the partner region which further provides the polyA signal. An additional DUX4 activation may result from relocation from a repressed to an actively 
transcribed nuclear compartment. Similar to the CIC-DUX4 chimeric transcript regulated by the CIC promoter in the $t(4 ; 19)$ translocation found in a Ewing-like sarcoma, the expression of the EWSR1-DUX4 fused gene in the RMS with a $\mathrm{t}(4 ; 22)$ translocation probably results from the activity of the EWSR1 promoter [3]. In other cancers, additional abnormalities of DUX4 have been observed including epigenetic alterations within the 4q35 FSHD-associated locus. Triggered by those observations, expression profiles compared FSHD and 35 different cancers revealing a significant level of similarity between FSHD and Ewing-like sarcomas [9].

\section{$D U X 4$ domains and functional predictions}

The N-terminal part of the 424 aminoacid-long DUX4 protein harbors two homeodomains and three nuclear localization sequences (NLS). The C-terminal domain contains a domain of interaction with the histone acetyltransferase p300/CBP and two distinct transcription regulation signals required for DUX4-induced cytotoxicity. Thus, the CIC- and EWSR1-DUX4 fusion proteins should combine the DNA-binding specificity of CIC/EWSR1 with the transcriptional regulation and p300/CBPbinding capacities of DUX4. Based on experimental observations, truncation or replacement of the C-terminus should reduce/modify the transcriptional activation capacity of DUX4 [10] and inhibit interaction with p300/CBP. Indeed, the C-terminus truncated, but not the full-length copy, of DUX4 induced leukemic transformation rather than apoptosis [4].

\section{Pathological consequences of $D U X 4$ expression}

In FSHD myotubes, an overexpression of DUX4 induces cellular atrophy via activation of MuRF1 and MAFbx/atrogin-1, two E3 ubiquitin ligases and apoptosis via caspase 3 and p53. DUX4 deregulates myogenic differentiation by decreasing $M Y O D$ expression and induces aberrant expression of genes normally expressed specifically in germ cells. DUX4 expression additionally leads to production of reactive oxygen species (ROS) and DNA damage [11,12]. In cancer, DUX4induced ROS may induce mutations and chromosomal aberrations that contribute to malignant transformation (Figure 1D). Significantly, FSHD cells show transcriptional profiles similar to some cancer cells [9]. In leukaemia cells, DUX4 induces expression of ERG dominant negative isoforms resulting in the loss of function of wild-type ERG which is essential for leukemogenesis [13]. In nude mice expressing IGH-DUX4 but not DUX4 in pro-B cells generated a B-cell leukaemia suggesting that DUX4 gains an oncogenic potential following chromosomal rearrangement [1]. In the BCP-ALL subtype, DUX4-induced DNA damage can account for the frequent deletion of ERG and additional chromosomal aberrations which constitute a hallmark in this disease. In Ewing-like sarcoma, the CIC-DUX4 fusion results in changes in CIC transcriptional activity leading to 
upregulation of the PEA3 transcription factor that regulates many genes involved in oncogenesis [2].

\section{DUX4: therapeutic opportunities}

Several genetic and epigenetic approaches have been developed to inhibit DUX4 expression in the context of FSHD, including small interfering RNAs, antisense oligonucleotides, Morpholinos impeding polyadenylation or intron splicing, as well as microRNAs directly targeting DUX4. The promoter and exon 1 of DUX4 have been targeted by a dCas9-KRAB fusion protein, with KRAB significantly decreasing the expression levels of DUX4 and downstream genes [14]. Recently, compounds with epigenetic activity including some under test in clinical trials were used in screens to identify molecules that decrease or suppress DUX4 expression in FSHD myoblasts. Among different classes of molecules, inhibitors of the BET (bromodomain and extra-terminal) domain and beta-2 adrenergic receptor agonists have been identified [15]. Since aberrant expression of DUX4 fusion proteins induces leukemic transformation [4], some of these approaches could be potentially useful in cancers where DUX4 is rearranged and overexpressed.

\section{Concluding Remarks and Future Perspectives}

Numerous similarities exist in the transcriptional signatures of FSHD myoblasts and several cancers [9]. Recent discovery of the role of DUX4 in cancer provide new opportunities for both FSHD and cancer research and treatment. Cancer cells from patients with high expression of DUX4 fusion genes may provide human experimental models for DUX4-targeted drug screening. Vice versa, therapies developed for FSHD may prove of interest in cancer treatment. Future perspectives include the use of DUX4-targeting drugs developed for FSHD to silence its expression in cancer cells and possibly inhibit cancer cell growth. As DUX4 is not expressed in somatic tissues, it can be considered a "safe" target for cancer therapy.

\section{Acknowledgements}

The work was supported by grants from AFM (MEGAFSHD) to YV, from the Presidium of RAS and the State program of fundamental scientific research of IDB RAS to EK and YV, and from the Russian Foundation for Basic Research (No 19-04-01020) to EP.

\section{Disclaimer Statement}

The authors declare no conflict of interest. 


\section{Figure Legend}

Figure 1. DUX4 rearrangements in cancer $(A-C)$ and physiological consequences of its aberrant expression (D). (A), in Ewing-like sarcoma, the $\mathrm{t}(4 ; 19)(\mathrm{q} 35 ; \mathrm{q} 13)$ chromosomal translocation generates a fusion between the DUX4 C-terminus and the CIC gene. This fusion results in the production of CIC-DUX4 chimeric protein under the control of the CIC promoter; in ALL, translocation of a truncated or complete copy of $D U X 4$ into an intron of $E R G \mathrm{t}(4 ; 21)(\mathrm{q} 35 ; \mathrm{q} 22)(\mathbf{B})$ or into the $I G H$ locus $\mathrm{t}(4 ; 14)(\mathrm{q} 35 ; \mathrm{q} 32)$ (C) leads to expression of truncated or chimeric proteins. (D), pathological consequences of aberrant expression of DUX4 include transcription factors deregulation, apoptosis, leukemogenesis, oxidative stress, DNA damage and deregulation of myogenesis.

\section{Resources}

${ }^{\mathrm{i}}$ https://www.proteinatlas.org/ENSG00000260596-DUX4/pathology

\section{References}

1 Lilljebjörn, H. and Fioretos, T. (2017) New oncogenic subtypes in pediatric B-cell precursor acute lymphoblastic leukemia. Blood 130, 1395-1401

2 Kawamura-Saito, M. et al. (2006) Fusion between CIC and DUX4 up-regulates PEA3 family genes in Ewing-like sarcomas with $\mathrm{t}(4 ; 19)(\mathrm{q} 35 ; \mathrm{q} 13)$ translocation. Hum. Mol. Genet. 15, 2125-37

3 Sirvent, N. et al. (2009) Fusion of EWSR1 with the DUX4 facioscapulohumeral muscular dystrophy region resulting from $\mathrm{t}(4 ; 22)(\mathrm{q} 35 ; \mathrm{q} 12)$ in a case of embryonal rhabdomyosarcoma. Cancer Genet. Cytogenet. 195, 12-18

4 Yasuda, T. et al. (2016) Recurrent DUX4 fusions in B cell acute lymphoblastic leukemia of adolescents and young adults. Nat. Genet. 48, 569-574

5 Lemmers, R.J.L.F. et al. (2010) A unifying genetic model for facioscapulohumeral muscular dystrophy. Science (80-. ). 329, 1650-1653

6 Dixit, M. et al. (2007) DUX4, a candidate gene of facioscapulohumeral muscular dystrophy, encodes a transcriptional activator of PITX1. Proc Natl Acad Sci U S A 104, $18157-18162$

7 Petrov, A. et al. (2008) A nuclear matrix attachment site in the 4q35 locus has an enhancer-blocking activity in vivo: implications for the facio-scapulo-humeral dystrophy. Genome Res. 18, 39-45

8 van Overveld, P.G. et al. (2003) Hypomethylation of D4Z4 in 4q-linked and non-4q- 
linked facioscapulohumeral muscular dystrophy. Nat Genet 35, 315-317

9 Dmitriev, P. et al. (2014) Cancer-related genes in the transcription signature of facioscapulohumeral dystrophy myoblasts and myotubes. J Cell Mol Med 18, 208-217

10 Tanaka, Y. et al. (2018) Transcriptional activities of DUX4 fusions in B-cell acute lymphoblastic leukemia. Haematologica 103, e522-e526

11 Dmitriev, P. et al. (2016) DUX4-induced constitutive DNA damage and oxidative stress contribute to aberrant differentiation of myoblasts from FSHD patients. Free Radic. Biol. Med. 99, 244-258

12 Bosnakovski, D. et al. (2008) An isogenetic myoblast expression screen identifies DUX4-mediated FSHD-associated molecular pathologies. EMBO J 27, 2766-2779

13 Dong, X. et al. (2018) Structural basis of DUX4/IGH-driven transactivation. Leukemia $32,1466-1476$

14 Himeda, C.L. et al. (2018) Identification of Epigenetic Regulators of DUX4-fl for Targeted Therapy of Facioscapulohumeral Muscular Dystrophy. Mol. Ther. 26, 17971807

15 Campbell, A.E. et al. (2017) BET bromodomain inhibitors and agonists of the beta-2 adrenergic receptor identified in screens for compounds that inhibit DUX4 expression in FSHD muscle cells. Skelet. Muscle 7, 16 
A

\section{CIC-DUX4}

fusion

(Ewing-like

Sarcoma)
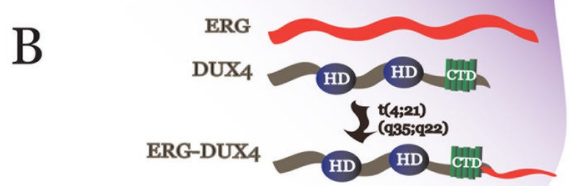

C
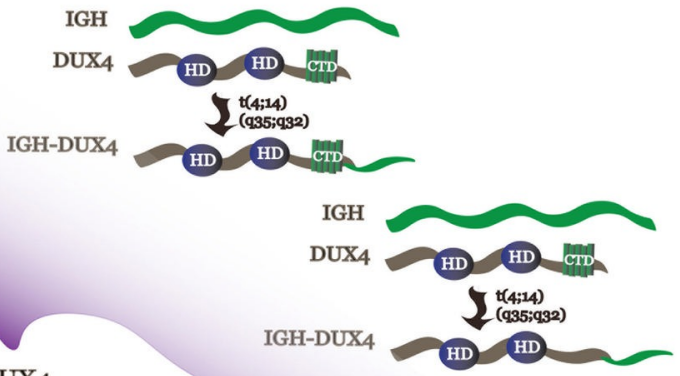

\section{IGH-DUX4}

(Acute

Lymphoblastic

Leukemia)

D

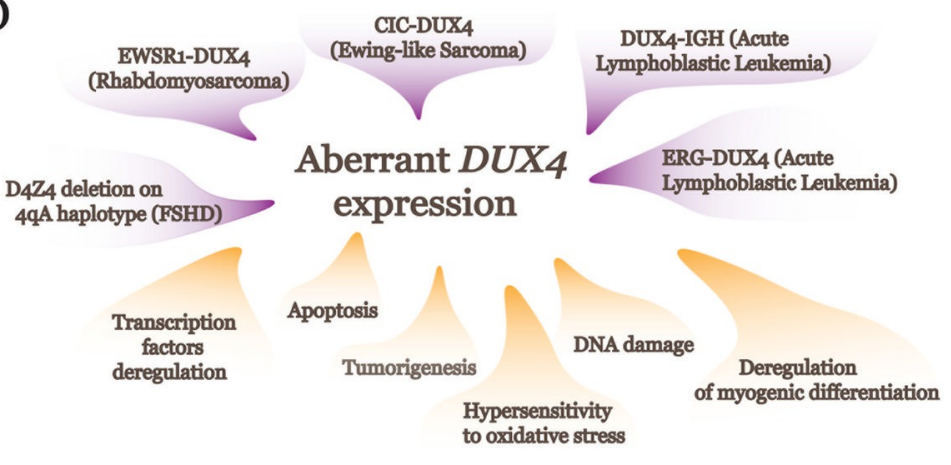

